Gruby, A. R. \& Few, A. V. (1959) J. gen. Microbiol. 20, 321-327

\title{
Osmotic Properties of Protoplasts of Micrococcus lysodeikticus
}

\author{
By A. R. GILBY* aNd The LAte A. V. FEW \\ Department of Colloid Science, University of Cambridge
}

SUMMARY: Protoplasts were released from Micrococcus lysodeikticus by the action of lysozyme in media containing sucrose, $\mathrm{NaCl}$ or mixtures of these. Empirical corrections by two methods were made for the effect of refractive index on the optical density of the protoplast suspensions measured at $500 \mathrm{~m} \mu$. With the assumption, valid for some similar systems, that the optical density of a protoplast suspension is inversely proportional to the protoplast volume, the protoplasts were shown to behave as osmometers at osmotic pressures above the threshold for lysis and to follow a van't Hoff-Boyle law. The osmotically-inactive volume in a standard stabilizing medium containing $\mathrm{M}$-sucrose $+0.05 \mathrm{M}-\mathrm{NaCl}$ (osmotic pressure $37.8 \mathrm{~atm}$.) was $c .76 \%$. Protoplasts formed in hypotonic media were more resistant to osmotic explosion than those formed at higher medium concentrations and subsequently diluted.

A cell which lacks structural rigidity will respond to changes in the osmotic pressure of its environment in a manner determined by the selective permeability properties of the external membrane. When the membrane is impermeable to an extracellular solute but freely permeable to the solvent the volume changes produced by osmosis in a system exposed to media of different osmotic pressure should follow the van't Hoff-Boyle law:

$$
\pi\left(V-V_{0}\right)=\text { constant, }
$$

where $\pi$ is the osmotic pressure of the medium, $V$ is the volume of the cell and $V_{0}$ is that volume of the cell which does not respond to osmotic influences. This law is followed by the cells of many higher organisms (Lucke, 1940). If, as suggested by recent work (McQuillen, 1955; Mitchell \& Moyle, 1956 $a, b$; Gilby, 1957), the protoplasts of Micrococcus lysodeikticus are surrounded by an osmotic membrane of low mechanical strength, these too should behave similarly.

Protoplasts of Bacillus anthracis and $B$. megaterium have been studied previously by using measurements based on microscopy. Stähelin (1954) investigated $B$. anthracis in sucrose and in $\mathrm{NaCl}$ solutions by qualitative methods. Weibull (1955) showed that protoplasts of $B$. megaterium followed equation (1) in sucrose, glucose and polyethylene glycol, but not in urethane. In the present investigation it will be shown that protoplasts of Micrococcus lysodeikticus also behave as osmometers in sucrose and $\mathrm{NaCl}$ solutions. The method evolved uses the optical properties of the protoplast suspensions and avoids some of the experimental difficulties of direct measurement. However, in its present development it does not permit the calculation of absolute volumes.

* Present address : Division of Entomology, C.S.I.R.O., Canberra, Australia. 


\section{METHODS}

Organism used. Micrococcus lysodeikticus (NCTC 2665) was grown on a beef tryptic digest agar medium at $\mathrm{pH} \mathbf{7 \cdot 2}$ in 20 oz. bottles. Each bottle was inoculated with $c .20 \mathrm{ml}$. of a $48 \mathrm{hr}$. broth culture and the organisms harvested in distilled water after c. $20 \mathrm{hr}$. incubation at $37^{\circ}$. After passage through a coarse glass filter, the bacteria were washed three times with distilled water by centrifugation.

Protoplast formation. Protoplasts were prepared from Micrococcus lysodeikticus by treatment with lysozyme in either of two ways. In some experiments, the organisms were suspended at a concentration equiv. to $0.5 \mathrm{mg}$. dry wt./ml. in either $\mathrm{NaCl}$ up to $2 \mathrm{M}$ or sucrose up to $2 \mathrm{M}$ containing $0.05 \mathrm{M}-\mathrm{NaCl}$, and then treated with lysozyme, $10 \mu \mathrm{g} . / \mathrm{ml}$. for 30-60 min. In other cases, protoplasts were prepared by reacting the organisms at equiv. $10 \mathrm{mg}$. dry wt./ $\mathrm{ml}$. in $\mathrm{M}$-sucrose $+0.05 \mathrm{M}-\mathrm{NaCl}$, with lysozyme $100 \mu \mathrm{g} . / \mathrm{ml}$. for $30 \mathrm{~min}$. These suspensions were subsequently diluted to be equiv. $0.5 \mathrm{mg}$. dry wt. organism/ ml. in media which yielded final compositions of either $0.05 \mathrm{M}-\mathrm{NaCl}$ with various concentrations of sucrose, $0.05 \mathrm{M}$-sucrose with various concentrations of $\mathrm{NaCl}$, or 0.5 M-sucrose with various concentrations of $\mathrm{NaCl}$. Protoplast membranes were prepared by the method previously described (Gilby, Few \& McQuillen, 1958) and suspended at equiv. $1 \mathrm{mg}$. membrane dry wt. $/ \mathrm{ml}$. in $\mathrm{NaCl}$ solutions up to $2 \mathrm{M}$.

Spectrophotometric measurements. The optical densities of the protoplast and membrane suspensions were measured at $500 \mathrm{~m} \mu$ with a Unicam SP 600 spectrophotometer.

\section{RESULTS}

The values of the optical density, measured at $500 \mathrm{~m} \mu$, of the various protoplast suspensions are plotted in Fig. 1 as a function of the osmotic pressure of the medium. The osmotic pressure of the standard stabilizing medium $1 \mathrm{M}$-sucrose $+0.05 \mathrm{M}-\mathrm{NaCl}$ used in previous work (Gilby \& Few, $1957 a, b$ ) is $\mathbf{3 7 . 8} \mathrm{atm}$. The results confirm that this is above the threshold for osmotic lysis. The sigmoid shape of the curves in the region of rapid change is a reflexion of the distribution of stability and of osmotic properties in the protoplast population, evident also in the size variation of protoplasts observed at fixed osmotic pressure in phase-contrast micrographs (Gilby, 1957). In contrast, both protoplast 'ghosts' and intact bacteria are more uniform in size. At osmotic pressures where the protoplasts are stable, the optical density of the suspensions, with the exception of those in pure sucrose, increases as the osmotic pressure increases. This is attributed to a decrease in volume of protoplasts. It is not known whether crenation occurs. With sucrose solutions, the increase in refractive index masks this effect. The differences between the curves at higher osmotic pressures are due to refractive index effects.

In order to use the present results to determine whether Micrococcus lysodeikticus protoplasts follow equation (1), it is necessary both to eliminate the effect on the optical density of the different refractive indices of the media and to relate the optical density to the average volume of the protoplasts. 
There is no theory of light scattering applicable to particles the size of protoplasts $(0 \cdot 5-1 \mu)$; hence it is necessary to rely on empirical methods.

Corrections for refractive index of medium. Two methods were used.

Method $\boldsymbol{A}$. From the known composition of each medium its refractive index was ascertained from the literature and, in many cases, checked by

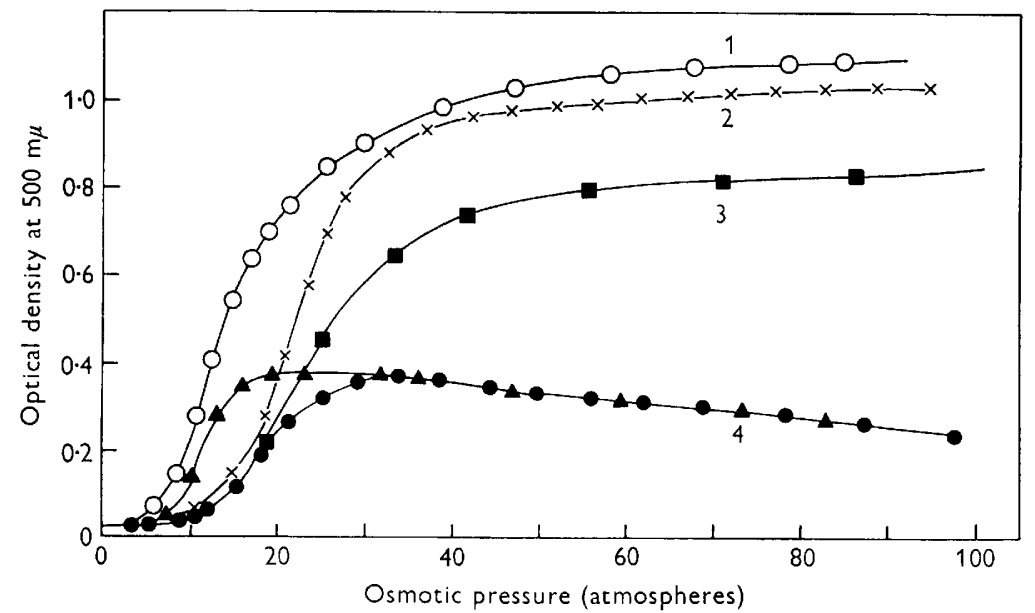

Fig. 1. Optical density of protoplasts of Micrococcus lysodeikticus suspended in solutions of different osmotic pressure. Protoplast suspensions at equiv. $0.5 \mathrm{mg}$. dry wt. $/ \mathrm{ml}$. in (1) $\mathrm{NaCl}$, (2) $\mathrm{NaCl}+0.05 \mathrm{M}$-sucrose, (3) $\mathrm{NaCl}+0.5 \mathrm{M}$-sucrose, (4) sucrose + 0.05 $\mathrm{M}-\mathrm{NaCl}$. $\Delta$, Protoplasts formed in test medium; $O$, protoplasts preformed in $\mathrm{M}$-sucrose $+\mathbf{0 . 0 5} \mathrm{M}$ $\mathrm{NaCl}$ and transferred to test medium.

measurement with an Abbé refractometer. The results in Fig. 1 were then used to plot, at fixed values of osmotic pressure, the optical density as a function of $\Delta n$, the difference in refractive index of the medium and water. $\mathbf{A}$ smooth curve was drawn through the points and extrapolated to estimate the optical density in a medium of refractive index equal to water.

Method $B$. This was based on the results in sodium chloride alone. Figure 2 shows the optical density of protoplast membranes suspended in solutions of $\mathrm{NaCl}$. At the lowest concentrations the membranes are unstable (Gilby et al. 1958). The curve in the stable region was therefore extrapolated to estimate the optical density of the membranes in water. It has been assumed that the different optical densities in Fig. 2 arise from the effect of the refractive index of the medium and that, in this regard, protoplast membranes and protoplasts behave similarly. At each concentration (osmotic pressure) of $\mathrm{NaCl}$, the ratio for membranes of the optical density in the medium to that in water was applied to correct the results with protoplasts to correspond to a medium of the refractive index of water.

Application of method A gave curve (1) of Fig. 3; results with Method B were so similar that a separate plot is not warranted.

Quantitative test of behaviour of protoplasts as osmometers. In the treatment of the results which follows, it is assumed that the optical density of a protoplast suspension is inversely proportional to the average protoplast volume. 


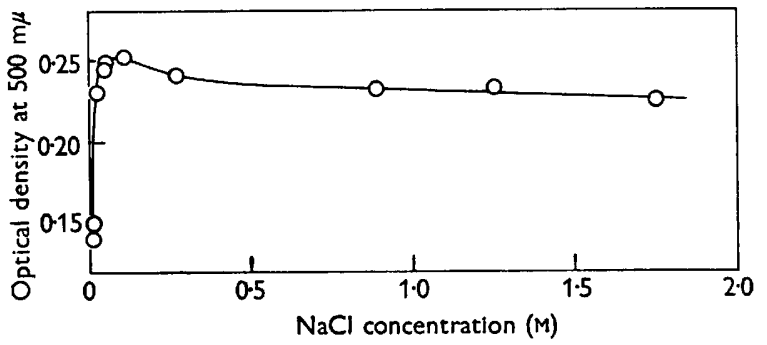

Fig. 2. Optical density of protoplast membranes of Micrococcus lysodeikticus suspended in

NaCl. Protoplast membranes at equiv. $1 \mathrm{mg}$. dry wt. membrane/ml.

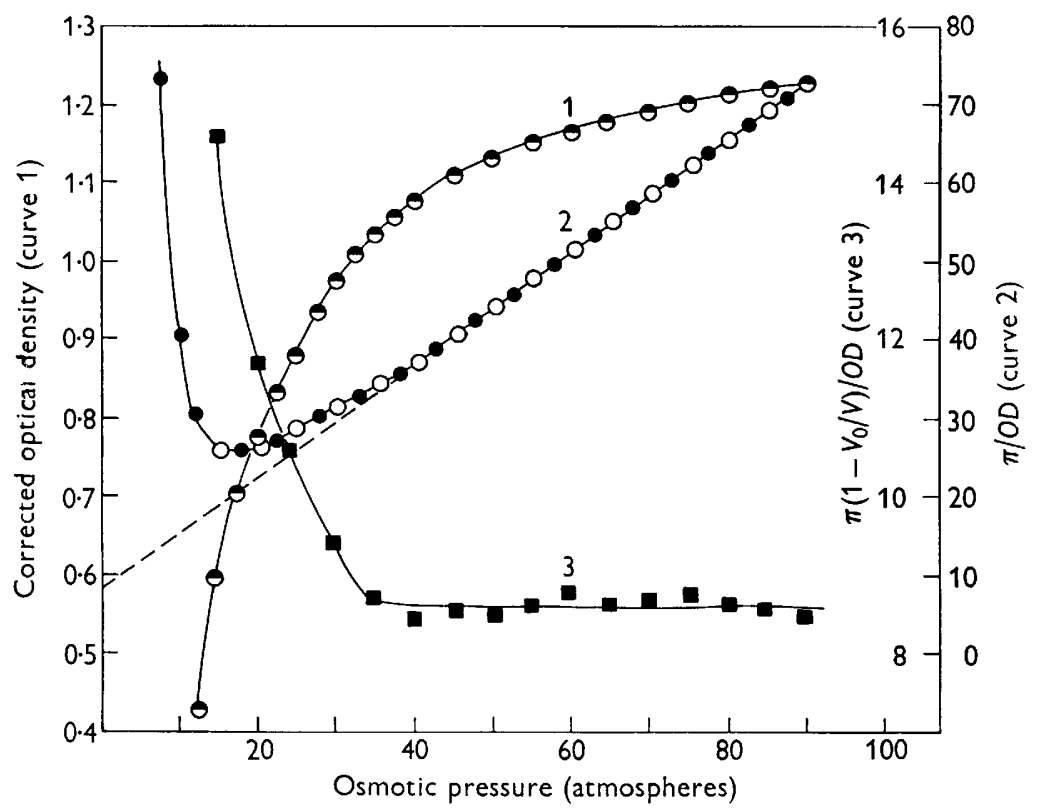

Fig. 3. Optical properties of protoplasts of Micrococcus lysodeikticus as a function of osmotic pressure. (1) Optical density at $500 \mathrm{~m} \mu$ corrected for refractive index of medium (method A). (2) Test of equation (3). Plot of $\pi / O D$ against $\pi$. O, Refractive index corrections by method $A$; $O$, refractive index corrections by method $B$. (3) Test of equation (4). Plot of $\frac{\pi}{O D}\left(1-\frac{V_{0}}{K} O D\right)$ against $\pi$.

Equation (1) may be written:

$$
\pi\left(V-V_{0}\right)=C,
$$

where $V$ and $V_{0}$ now refer to the volume of protoplasts in a suspension.

If $V=K / O D$ where $K$ is a constant and $O D$ is the optical density of a suspension at $500 \mathrm{~m} \mu$

then

$$
\frac{\pi}{O D}=\pi \frac{V_{0}}{K}+\frac{C}{K}
$$

Alternatively, equation (2) may be written

$$
\pi\left(1-\frac{V_{0}}{V}\right)=\frac{C}{V}
$$


therefore

$$
\pi\left(1-V_{0} \frac{O D}{K}\right)=C \frac{O D}{K}
$$

and

$$
\frac{\pi}{O D}\left(1-\frac{V_{0}}{K} O D\right)=\frac{C}{K} \text {. }
$$

From equation (3) it follows that, provided the protoplasts follow a van't Hoff-Boyle Law expressed by equation (1) and the assumptions detailed are valid, a plot of $\pi / O D$ against $\pi$ should be linear. Such a plot is shown in curve (2) of Fig. 3. The two symbols refer to calculations using method A or method B to correct for refractive index and these indicate perfect agreement. It is apparent that the results fit equation (3) for osmotic pressures greater than $30 \mathrm{~atm}$. At lower osmotic pressures the results deviate because of lysis of protoplasts. The slope of the linear portion is equal to $V_{0} / K$.

The value of $V_{0} / K$ obtained from the plot of equation (3) can then be inserted in equation (4). This has been done in curve (3) of Fig. 3 and the values of $\frac{\pi}{O D}\left(1-\frac{V_{0}}{K} O D\right)$ plotted against $\pi$. In conformity with equation (4), the values are constant at osmotic pressures greater than $30 \mathrm{~atm}$., the same range of validity as equation (3) from which $V_{0} / K$ was derived.

\section{DISCUSSION}

The experimental methods described possess some advantages, particularly in convenience and the use of a large population, over the method of direct measurement by microscope as used, for example, by Weibull (1955). However, the treatment of results depends on the assumption that the optical density of a protoplast suspension is inversely proportional to the protoplast volume. Because of their small size and fragility the experimental confirmation of this by methods such as haematocrit measurements is likely to prove difficult. Comparable information on other similar systems is rare. With erythrocytes it is sometimes found that the relative light transmission of a system decreases linearly as the cell volume increases. Over a wide range of optical densities, $\log I_{0} / I$ will then be inversely proportional to volume. Further, Wilbur \& Collier (1943) found that the product of the optical density and the volume of erythrocytes was constant over a wide range of $\mathrm{NaCl}$ concentrations. With rat liver mitochondria also, it was established empirically (Tedeschi \& Harris, 1955,1958 ) that the optical density of a suspension was inversely proportional to the mitochondrial volume. Although not quantitatively applicable to the present case because the particle size is too great and refraction may occur, Mie theory does predict that as the particle size becomes larger the optical density of a suspension can, under certain conditions, decrease (Heller \& Pangonis, 1954). Nevertheless, in the present case the final justification for the assumption lies in the low probability of obtaining the curves (2) and (3) in Fig. 3 in the event of the relation not holding.

The results outlined therefore support the conclusion that protoplasts of Micrococcus lysodeikticus behave as osmometers at osmotic pressures above the 
threshold for lysis. It follows that they must be readily permeable to water. In a medium of low osmotic pressure, the protoplasts imbibe water and, lacking mechanical rigidity and the restraint of the cell wall, they rupture. Since $\left(V_{0} / K\right) O D=V_{0} / V$, then the calculation of $V_{0} / K$ from equation(3) and Fig. 3, curve (2), as set out in the previous section, also enables the calculation of the ratio $V_{0} / V$ at any osmotic pressure for which the value of the optical density corrected for refractive index is known. For example, in the standard stabilizing medium of $\mathrm{M}$-sucrose $+0.05 \mathrm{M}-\mathrm{NaCl}$, the osmotically inactive volume is approximately $76 \%$ of the total protoplast volume. Until the value of $K$ is determined, and the basic assumption on the relation between volume and optical density thereby tested independently, it is not possible to give other than relative figures.

Weibull (1955) noted that although protoplasts of Bacillus megaterium followed equation (1) after formation, the size of freshly prepared protoplasts was independent of the osmotic pressure of the medium in which they were formed. This does not appear to be so with protoplasts of Micrococcus lysodeikticus at osmotic pressures above the stabilization threshold. Furthermore, the optical density of a suspension of $\boldsymbol{M}$. lysodeikticus protoplasts in a medium of given composition is the same (Fig. 1) whether the protoplasts have been formed in that medium or else have been prepared in the standard $1 \mathrm{M}$-sucrose medium and subsequently transferred to the test medium. However, at osmotic pressures below $30 \mathrm{~atm}$., there is evidence that the protoplasts in this substabilization range were more stable when formed in the medium than when transferred from M-sucrose. Apparently the protoplasts are more sensitive to a suddenly applied osmotic shock, or else may be able to adjust to their environment while losing their cell walls. These observations were not due to momentary exposure to grossly hypotonic solutions; this was rigorously avoided when changes in medium were being effected.

The surviving author wishes to express his thanks to the Commonwealth Scientific and Industrial Research Organization (Australia) for the award of an Overseas Studentship. The late A. V. Few was a member of the scientific staff of the Medical Research Council.

\section{REFERENCES}

Girby, A. R. (1957). The Physical Chemistry of Bacterial Protoplasts. Ph.D. Dissertation, University of Cambridge.

Girby, A. R. \& FEw, A. V. (1957a). The reactivity of ionic detergents with Micrococcus lysodeikticus. Nature, Lond. 179, 422.

GiLby, A. R. \& Few, A. V. (1957b). Surface chemical studies on the protoplast membrane of Micrococcus lysodeikticus. Proc. 2nd Int. Congr. Surface Activity, 4, 262. London: Butterworths Scientific Publishers.

Gilby, A. R., Few, A. V. \& McQuillen, K. (1958). The chemical composition of the protoplast membrane of Micrococcus lysodeikticus. Biochim. biophys. Acta, 29, 21.

Heller, W. \& Pangonis, W. J. (1954). Light scattering of spherical colloidal particles. J. chem. Phys. 22, 948.

Lucke, B. (1940). The living cell as an osmotic system and its permeability to water. Cold Spr. Harb. Symp. quant. Biol. 8, 123. 
McQuillen, K. (1955). Bacterial protoplasts, I. Protein and nucleic acid metabolism in protoplasts of Bacillus megaterium. Biochim. biophys. Acta, 17, 382.

Mrtchell, P. \& MoYle, J. (1956a). Osmotic function and structure in bacteria. In Bacterial Anatomy. Symp. Soc. gen. Microbiol. 6, 150.

Mitchell, P. \& Moyle, J. (1956b). Liberation and osmotic properties of the protoplasts of Micrococcus lysodeikticus and Sarcina lutea. J. gen. Microbiol. 15, 512.

StäHelin, H. (1954). Über osmotisches Verhalten und Fusion nackter Protoplasten von Bacillus anthracis. Schreiz. Z. allg. Path. 17, 296.

Tedeschi, H. \& Harris, D. L. (1955). The osmotic behaviour and permeability to non-electrolytes of mitochondria. Arch. Biochem. Biophys. 58, 52.

Tedeschi, H. \& Harris, D. L. (1958). Some observations on the photometric estimation of mitochondrial volume. Biochim. biophys. Acta, 28, 392.

WeIbUll, C. (1955). Osmotic properties of protoplasts of Bacillus megaterium. Exp. cell. Res. 9, 294.

Wilbur, K. M. \& Collier, H. B. (1943). A comparison of the haemolytic actions of lysolecithin and saponin. J. cell. comp. Physiol. 22, 233.

(Received 20 October 1958) 